\title{
Lipid profile and markers of bone health in free living older men and women
}

\author{
E. Laird ${ }^{1}$, M. Ward ${ }^{1}$, H. McNulty ${ }^{1}$ J. M. W. Wallace ${ }^{1}$, M. Healy ${ }^{2}$, M. C. Casey $^{2}$ and J. J. Strain ${ }^{1}$ \\ ${ }^{1}$ Northern Ireland Centre for Food and Health (NICHE) University of Ulster, Coleraine BT52 1SA, ${ }^{2}$ The Mercers Institute \\ for Research on Ageing (MIRA) and the Department of Biochemistry, St James's Hospital, Dublin, Ireland
}

Osteoporosis and osteopenia are increasingly common conditions associated with decreased bone mineral density (BMD) and increased bone fragility. Patients with atherosclerosis are at a higher risk of osteoporosis and specific lipid profiles have been associated with $\mathrm{BMD}^{(1,2)}$. The aim of this study was to assess the relationship between lipid profile and bone health in a sub-sample of participants $(n 955)$ from the TUDA (Trinity Ulster Department of Agriculture) cohort study. Participants were recruited based on the following criteria; aged $>60$ years, diagnosed hypertensive, born (or have parents born) on the Island of Ireland and no evidence of severe dementia. A lipid profile was measured and BMD of the total hip, femoral neck and lumbar vertebrae were measured using total body dual-energy X-ray absorptiometry (DXA) (Lunar Prodigy, GE Healthcare, UK). Individuals were classified as normal, osteopenic and osteoporotic using BMD T-scores $^{(3)}$ and also according to established cut-off values for high density lipoprotein (HDL) and low density lipoprotein (LDL) concentration $^{(4)}$. There were no significant associations between total cholesterol or LDL concentrations and BMD at any site (data not shown). The results were then analysed by HDL range ${ }^{(4)}$ (Table 1$)$.

Table 1. Baseline characteristics by HDL range ${ }^{4}$

\begin{tabular}{|c|c|c|c|c|}
\hline \multicolumn{5}{|c|}{ HDL range } \\
\hline & $\begin{array}{c}<1.03 \mathrm{mmol} / \mathrm{l} \\
n 210\end{array}$ & $\begin{array}{c}1.03-1.55 \mathrm{mmol} / \mathrm{l} \\
n 451\end{array}$ & $\begin{array}{c}>1.55 \mathrm{mmol} / \mathrm{l} \\
n 294\end{array}$ & $P$ \\
\hline Age (yrs) & $69.0(64.5,73.3)^{\mathrm{a}}$ & $69.6(65.5,73.8)^{\mathrm{a}}$ & $72.2(67.3,77.1)^{b}$ & $<0.001$ \\
\hline Sex & $24.8 \%$ Female & 49.7\% Female & $69 \%$ Female & \\
\hline BMI $\left(\mathrm{kg} / \mathrm{m}^{2}\right)$ & $30.1(28.0,32.8)^{\mathrm{a}}$ & $29.6(26.6,33.3)^{\mathrm{a}}$ & $27.5(24.8,31.2)^{b}$ & $<0.001$ \\
\hline Vitamin D (nmol/l) & $41.4(26.5,56.2)^{\mathrm{a}}$ & $42.6(29.4,60.0)$ & $48.1(31.1,71.9)^{\mathrm{b}}$ & $<0.05$ \\
\hline Total Hip BMD $\left(\mathrm{g} / \mathrm{cm}^{2}\right)$ & $1.0(0.88,1.1)$ & $0.9(0.8,1.0)$ & $0.9(0.8,1.0)$ & 0.07 \\
\hline Femoral neck BMD $\left(\mathrm{g} / \mathrm{cm}^{2}\right)$ & $0.9(0.8,1.0)^{\mathrm{a}}$ & $0.8(0.7,0.9)^{\mathrm{b}}$ & $0.8(0.7,0.9)^{\mathrm{b}}$ & $<0.05$ \\
\hline Vertebral BMD $\left(\mathrm{g} / \mathrm{cm}^{2}\right)$ & $1.2(1.0,1.3)^{\mathrm{a}}$ & $1.1(1.0,1.3)^{\mathrm{b}}$ & $1.0(0.9,1.2)^{\mathrm{c}}$ & $<0.001$ \\
\hline Total Hip T-score & $-0.3(-1.0,0.6)^{\mathrm{a}}$ & $-0.4(-1.2,0.4)^{\mathrm{a}}$ & $-1.0(-1.7,1.2)^{\mathrm{b}}$ & $<0.001$ \\
\hline Femoral neck T-score & $-1.0(-1.7,-0.2)^{\mathrm{a}}$ & $-1.0(-1.8,-0.3)^{\mathrm{a}}$ & $-1.4(-2.0,0.7)^{\mathrm{b}}$ & $<0.001$ \\
\hline Vertebral T-score & $0.1(-1.2,1.2)^{\mathrm{a}}$ & $-0.4(-1.5,0.9)^{\mathrm{b}}$ & $-1.1(-2.2,0.2)^{\mathrm{c}}$ & $<0.001$ \\
\hline
\end{tabular}

${ }^{5}$ Different superscript letters denote statistically significant differences $(P<0.05)$; Statistical significance was assessed using One-way

ANOVA with a post-hoc Tukey test.

Analysis showed that individuals with osteoporosis had a significantly higher concentration of HDL than those with osteopenia $(<0.01)$ or normal $(<0.01)$ BMD and those with the highest concentration of HDL had a significantly lower T-score at all three sites $(<0.001)$. These differences were primarily driven by the females (data not shown). We speculate that HDL may negatively impact on bone health possibly via decreasing the osteogenic differentiation of mesenchymal stem cells (MSCs) to osteoblasts as suggested previously ${ }^{(1)}$.

We would like to acknowledge the co-funding for this research from the Irish Department of Agriculture and the Northern Ireland Department for Employment and Learning through its "Strengthening the all-Island Research Base" initiative.

1. Buizert J, Van Schoor NM, Lips P, et al. J Bone Miner Res, (2009); 24(6): 1103-1109.

2. Reed R, Wise R, Dobs A, et al. Resp Med, (2010); 104(12): 1943-1950.

3. World Health Study Group (1994) Technical Report Series, No. 843.

4. National Heart, Lung, and Blood Institute (NHLBI) (2002); Third Report of the National Cholesterol Education Program (NCEP). 